Cite this: Phys. Chem. Chem. Phys. 2014, 16, 8384

Received 15th December 2013, Accepted 10th March 2014

DOI: $10.1039 / c 3 c p 55284 e$

www.rsc.org/pccp

\title{
IR spectroscopy of pyridine-water structures in helium nanodroplets
}

\author{
Pablo Nieto, ${ }^{\mathrm{ab}}$ Melanie Letzner, ${ }^{a}$ Torsten Endres, ${ }^{a}$ Gerhard Schwaab $^{\mathrm{a}}$ and \\ Martina Havenith ${ }^{\star a}$
}

\begin{abstract}
We present the results of an IR spectroscopic study of pyridine-water heterodimer formation in helium nanodroplets. The experiments were carried out in the frequency range of the pyridine $\mathrm{C}-\mathrm{H}$ stretch region $\left(3055-3100 \mathrm{~cm}^{-1}\right)$ and upon water deuteration in the D-O stretch region $\left(2740-2800 \mathrm{~cm}^{-1}\right)$. In order to come to an unambiguous assignment we have determined the angle between the permanent dipole and the vibrational transition moment of the aggregates. The experiments have been accompanied by theoretical simulations which yielded two minimum structures with a $16.28 \mathrm{~kJ} \mathrm{~mol}^{-1}$ energy difference. The experimentally observed bands were assigned to two structures with different $\mathrm{H}$-bonds: an $\mathrm{N} \cdots \mathrm{H}$ bond and a bifurcated $\mathrm{O} \ldots \mathrm{H}-\mathrm{C}$ bond.
\end{abstract}

\section{Introduction}

The hydrogen bond as an example of noncovalent interaction is one of the most extensively studied subjects in physics, chemistry and biology. ${ }^{1-7}$ It plays a central role in the structure and chemistry of molecules of biological importance including peptides, proteins and DNA. In general, $\mathrm{CH} \cdots \pi, \mathrm{CH} \cdots \mathrm{lp}$, and $\pi \cdots \pi$ interactions are important for aggregation of different kinds of biomolecules. We decided to study the pyridine-water heterodimer as a model system for the competition between $\mathrm{CH} \cdots \mathrm{O}$ and $\mathrm{N} \cdots \mathrm{H}$ interaction. Pyridine and water have, in principle, two possible configurations for hydrogen bond formation. The first one is formed by bonding the $\mathrm{H}$ atom of the water molecule and the $\mathrm{N}$ atom of the pyridine ring. The second one is determined by the interaction between oxygen and the $\mathrm{C}-\mathrm{H}$ groups. In the following discussion we will refer to these cases as the $\mathrm{NH}$ bonded and the $\mathrm{OH}$ bridge structures, respectively. The $\mathrm{NH}$ bonded aggregate was predicted to be the global minimum by $a b$ initio calculations at the HF/STO-3G level of theory. ${ }^{8}$ This structure has been observed in previous noble gas matrix isolation studies by means of IR spectroscopy. ${ }^{9}$ In none of the previous theoretical studies has the $\mathrm{OH}$ bridge been predicted to be a stable structure. However, for a similar system, the pyrimidine-water heterodimer, Howard et al. predicted a local minimum for a $\mathrm{C}-\mathrm{H} \cdots \mathrm{O}$ bonded configuration. ${ }^{10}$

Helium nanodroplets provide the ideal matrix for aggregation and stabilization of a variety of weakly bonded complexes

\footnotetext{
${ }^{a}$ Department of Physical Chemistry II, Ruhr-Universität Bochum, Universitätsstraße 150, D-44780 Bochum, Germany. E-mail: martina.havenith@rub.de

${ }^{b}$ Institut für Optik und Atomare Physik, Technische Universität Berlin,

Hardenbergstraße 36, 10623 Berlin, Germany
}

as a result of their unique properties. ${ }^{11,12}$ The successive pick-up process permits fast thermalization of the molecules to the nanodroplet equilibrium temperature $(0.37 \mathrm{~K})$ before aggregation. ${ }^{13}$ Furthermore, due to the weak interaction between the helium and the embedded molecules, a very small matrix-induced shift for vibrational transitions, typically less than a few wavenumbers, is observed. These properties have triggered the use of helium nanodroplets in spectroscopic studies of atoms, molecules and clusters at sub-kelvin temperatures. It is now well established that the aggregation process is in many cases kinetically and not thermodynamically controlled, especially for molecules with large permanent electric dipole moments. This allowed the observation of local energy minima. ${ }^{4,14,15}$ The observation of structures, which correspond to local rather than global minima, has been explained by the electrostatic steering mechanism. The dipole-dipole interaction between pre-aligned monomers follows $\mathrm{a} \mathrm{r}^{-3}$ dependence and is thus the dominating interaction at a long range for neutral molecules with a permanent dipole moment. At low temperatures, higher rotational levels are usually not populated, which results in an effective orientation of the molecules prior to aggregation. Therefore, when the molecules approach one another the formation of dipole-dipole aligned structures is favored. When the molecules aggregate, the aggregation energy is dissipated very effectively by the liquid helium. At a temperature of $0.37 \mathrm{~K}$ the molecular aggregate might get trapped in a local minimum. This aggregation process is able to explain the head-to-tail configurations observed for molecules with high dipole moment such as HCN and formic acid, corresponding to a local, rather than a global, minimum of the potential energy surface. In the case of pyridine-water, the dipole moment of both monomers is large $\left(2.22 \mathrm{D}\right.$ for pyridine ${ }^{16}$ and $1.85 \mathrm{D}$ for water $^{17}$ ). For both, $\mathrm{NH}$ bonded and $\mathrm{OH}$ bridge 
structures, the dipole moments of the two monomers are oriented in line. As a consequence, the electrostatic steering can favor the formation of both types of hydrogen bonded structures.

We present the results of pyridine-water heterodimer formed in helium nanodroplets. In the experiments, the $\mathrm{C}-\mathrm{H}$ stretch region for the pyridine- $\mathrm{H}_{2} \mathrm{O}$ and the $\mathrm{D}-\mathrm{O}$ stretch region for the case of the pyridine- $\mathrm{D}_{2} \mathrm{O}$ complexes were scanned. As additional information, the vibrational transition moment angle VTMA was determined by means of a DC electric field. Comparison with $a b$ initio calculations revealed that both the $\mathrm{NH}$ bonded and $\mathrm{OH}$ bridge structures are formed in our experiments.

\section{Methods}

\subsection{Experimental methods}

We have studied the IR-absorption of pyridine-water heterodimers with the Bochum helium nanodroplet apparatus using an IR-OPO laser as a radiation source. A brief description of the experimental set-up, which is displayed schematically in Fig. 1, will be given in this section. Further details are given elsewhere. ${ }^{18}$ The helium nanodroplets were formed by supersonic expansion of ultra-pure helium gas (Alphagaz 2, Air Liquide) through a $5 \mu \mathrm{m}$ diameter nozzle into a vacuum. The temperature of the nozzle was kept at $14.5 \mathrm{~K}$ using a PID controller in a continuous flow liquid helium cryostat. At a stagnation pressure of 55 bar, droplets with an estimated average cluster size of 16000 atoms were formed. ${ }^{19}$ The stagnation conditions were obtained by optimizing the signal-to-noise ratio. The helium nanodroplets passed through a so-called pick-up chamber where additional molecules are present. The molecules are picked up by the superfluid helium nanodroplet and cooled to $0.37 \mathrm{~K}$ prior to aggregation. In the pick-up chamber, the partial pressures of pyridine and $\mathrm{H}_{2} \mathrm{O}$ were adjusted by computer controlled leak valves (Leybold MOVE 1250). The purity and partial pressures of the dopants were recorded by means of a residual gas analyzer (Pfeiffer PrismaPlus QMG 220). The ion currents for the $\mathrm{m} / \mathrm{z}$ channels of interest were monitored during the experiments.

The IR laser beam was amplitude-modulated by a chopper. It was coupled in from the backside, thereby enabling an optimal overlap with the helium droplet beam (antiparallel configuration) or from the side using a multipass cell (perpendicular configuration). The gold coated mirror set-up could also be used for the alignment of molecules in a DC electric field. A large DC electric field of up to $53 \mathrm{kV} \mathrm{cm}^{-1}$ can be applied in the laser interaction zone between two electrodes which are positioned perpendicular to the multipass cell. The laser polarization was adjusted to be either parallel or perpendicular to the DC electric field. Droplet depletion by evaporative cooling was detected using a Pfeiffer QMS 422 quadrupole mass spectrometer, which was operated in high pass filter mode with the lowest mass set to $8 \mathrm{amu}$. The signal from the mass spectrometer was demodulated using a lock-in amplifier to record the light-induced beam depletion. Infrared radiation was generated using a home-built continuous wave optical parametric oscillator based on a $\mathrm{MgO}$ doped periodically poled lithium niobate (PPLN) crystal, which is described in detail elsewhere. ${ }^{20}$ The OPO is pumped using a Nd:YAG master oscillator (Lightwave-Electronics model no. 126-1064-500) power amplifier system, providing an output power of typically $14 \mathrm{~W}$ at $1064 \mathrm{~nm}$. The PPLN crystal is enclosed in an oven $\left(30-200{ }^{\circ} \mathrm{C}\right)$, where the temperature is kept constant within $0.01{ }^{\circ} \mathrm{C}$, to maintain quasi-phase matching

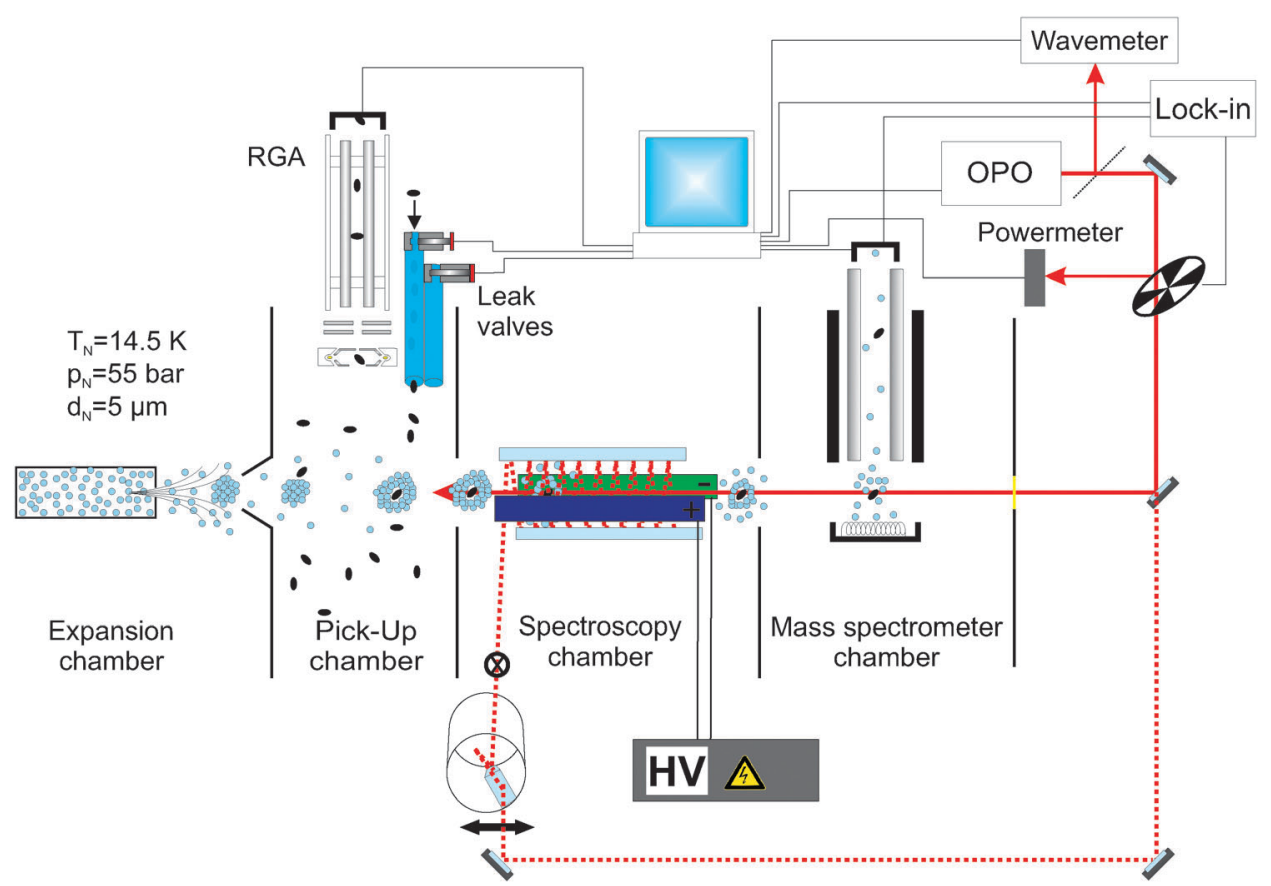

Fig. 1 Set-up of the Bochum helium nanodroplet spectroscopy experiment. The laser can be coupled in either antiparallel (red solid line) or perpendicular (red dashed line) to the helium nanodroplet beam. 
conditions. In the crystal, the pump wave is split into a signal and an idler wave. The idler wave has a maximum output power of $1.7 \mathrm{~W}$, and a linewidth of $10^{-4} \mathrm{~cm}^{-1}$. The laser is tunable between 2600 and $3300 \mathrm{~cm}^{-1}$. It can be continuously tuned over a range of $1.5 \mathrm{~cm}^{-1}$ before a $6.78 \mathrm{~cm}^{-1}$ free spectral range intracavity etalon has to be tilted for frequency adjustment. The idler wavelength is monitored using a Bristol Instruments wavemeter (model no. 621A-IR). The absolute frequency calibration has an accuracy of $0.1 \mathrm{~cm}^{-1}$, while the relative frequency calibration and the spectral resolution are much more precise and only limited by the linewidth of the laser.

\subsection{Theoretical methods}

In order to assign the experimentally observed bands, we have carried out accompanying $a b$ initio calculations using the TURBOMOLE suite of programs. ${ }^{21}$ As a first step, starting configurations were randomly generated and used as an input for a DFT optimization. For this initial screening, the b97 functional with dispersion correction $^{22}$ and the aug-cc-pVTZ basis set were used. More than a thousand structures were generated and subsequently optimized to ensure an extensive sampling of the configurational space. After optimization of 320 initial configurations a further search did not yield anymore new minima. In the following, only optimized structures with binding energies higher than $2.0 \mathrm{~kJ} \mathrm{~mol}^{-1}$ were taken into consideration. Subsequently, RI-MP2/aug-cc-pVTZ calculations were carried out on the DFT optimized structures. As a result, we found two stable conformations. Vibrational spectra were calculated by harmonic frequency analysis. Separate calculations were carried out for pyridine and $\mathrm{D}_{2} \mathrm{O}$ monomers in order to deduce scaling factors.

\section{Results and discussion}

\subsection{Theoretical structures}

The two stable structures of the pyridine-water heterodimer are displayed in Fig. 2. For the global minimum shown in Fig. 2(a) the $\mathrm{NH}$ bonded structure - a binding energy of $31.72 \mathrm{~kJ} \mathrm{~mol}^{-1}$ a)
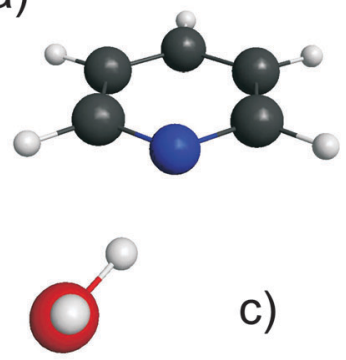

c) b)
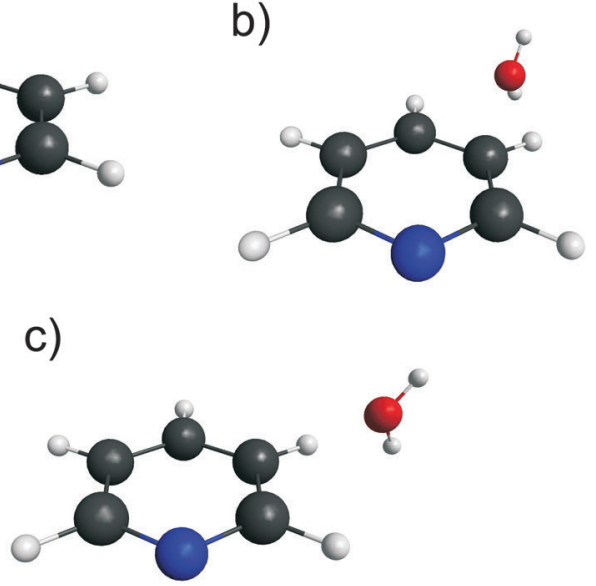

Fig. 2 Conformations obtained from ab initio calculations: (a) $\mathrm{NH}$ bonded structure (b) $\mathrm{OH}$ bridge structure (c) transition state. was obtained at the RI-MP2/aug-cc-pVTZ level of theory. This was corrected to $24.06 \mathrm{~kJ} \mathrm{~mol}^{-1}$ taking into account zero point energy (ZPE) correction. As a second stable heterodimer the $\mathrm{OH}$ bridge structure is shown in Fig. 2(b). A binding energy of $10.75 \mathrm{~kJ} \mathrm{~mol}^{-1}$ was obtained for this structure, $7.78 \mathrm{~kJ} \mathrm{~mol}^{-1}$ with ZPE correction.

In a previous Ar-matrix FT-IR spectroscopy study of this system, the spectra were assigned to the $\mathrm{NH}$ bonded structure. ${ }^{23}$ The binding energy for the complex was calculated to be $31.76 \mathrm{~kJ} \mathrm{~mol}^{-1}$, at the MP2/6-31++G** level of theory. However, no further structures for the heterodimer were considered in this work nor in any other previous studies on pyridine-water complexes. Whereas the $\mathrm{OH}$ bridge structure has not been considered for the pyridine-water dimer, a structure similar to the $\mathrm{OH}$ bridge structure was predicted as a local minimum for the pyrimidine-water heterodimer. ${ }^{10}$ In this case, the oxygen is bonded to a single hydrogen of the pyrimidine ring instead of being placed in the bridge position. It should be noted that in the first optimization step using DFT, additional structures were found with the $\mathrm{O}$ atom pointing towards the different hydrogens of the pyridine ring. However, when optimizing these conformations at the RI-MP2/aug-cc-pVTZ level, all structures besides the $\mathrm{OH}$ bridge structure shown in Fig. 2(b) relaxed to the $\mathrm{NH}$ bonded structure. Interestingly, the same was found to be the case in the theoretical work on pyrimidine-water by Howard et al. ${ }^{10}$

A search for the transition state for the two minima found at the RI-MP2/aug-cc-pVTZ level of theory was carried out. The structure is displayed in Fig. 2(c). The bonding energy for the transition state was found to be $9.54 \mathrm{~kJ} \mathrm{~mol}^{-1}\left(6.90 \mathrm{~kJ} \mathrm{~mol}^{-1}\right.$ with ZPE correction). Hence, the barrier between both heterodimers amounts to only $0.88 \mathrm{~kJ} \mathrm{~mol}^{-1}$.

The results of the predictions for the IR bands of the $\mathrm{NH}$ bounded and the $\mathrm{OH}$ bridge structures are summarized in Table 1 . When replacing $\mathrm{H}_{2} \mathrm{O}$ by $\mathrm{D}_{2} \mathrm{O}$, contamination by HDO produced by a fast exchange reaction between $\mathrm{H}_{2} \mathrm{O}$ and $\mathrm{D}_{2} \mathrm{O}$ in the inlet gas lines was unavoidable. In principle, both pyridine$\mathrm{D}_{2} \mathrm{O}$ and pyridine-HDO dimers could be observed in our experiments. Therefore, we have also calculated the D-O stretch band frequencies for pyridine-HDO. In the case of pyridineHDO either the $\mathrm{D}$ atom or the $\mathrm{H}$ atom can point towards the nitrogen atom of pyridine, with the $\mathrm{D}-\mathrm{O}$ stretch transition being predicted at $2514.5 \mathrm{~cm}^{-1}$ or at $2735.0 \mathrm{~cm}^{-1}$, respectively for the $\mathrm{NH}$ bonded structure.

In the case of pyridine- $\mathrm{H}_{2} \mathrm{O}$, all band frequencies have been multiplied by the same scaling factor. We have predicted the spectrum of the pyridine monomer at the same level of theory. The scaling factor was deduced by comparison to the experimentally observed $\mathrm{C}-\mathrm{H}$ stretch band at $3087.66 \mathrm{~cm}^{-1}$. This yielded a scaling factor of 0.988 for DFT and 0.957 for MP2. Predictions for the D-O stretches for the pyridine- $\mathrm{D}_{2} \mathrm{O}$ were obtained in a similar way. Here, the comparison to the experimentally observed $\mathrm{D}_{2} \mathrm{O}$ asymmetric stretch band at $2798.58 \mathrm{~cm}^{-1}$ yielded a scaling factor of 0.997 for DFT and of 0.968 for the MP2 calculations.

\subsection{C-H stretch spectral range with pyridine and $\mathrm{H}_{2} \mathrm{O}$}

As a first experimental step, we investigated the $\mathrm{C}-\mathrm{H}$ stretch in the frequency range between 3055 and $3100 \mathrm{~cm}^{-1}$. The pyridine 
Table 1 Predicted central band frequencies $\left(\mathrm{cm}^{-1}\right)$, intensities $\left(\mathrm{km} \mathrm{mol}^{-1}\right.$ in brackets) and VTMA for stable structures for DFT and MP2 calculations. Predicted frequencies for the $\mathrm{C}-\mathrm{H}$ stretches are given in the left column. Predicted frequencies for pyridine $-\mathrm{D}_{2} \mathrm{O}$ and pyridine- $\mathrm{HDO}$ complexes in the frequency range between 2720 and $2800 \mathrm{~cm}^{-1}$ (D-O stretches) are also listed

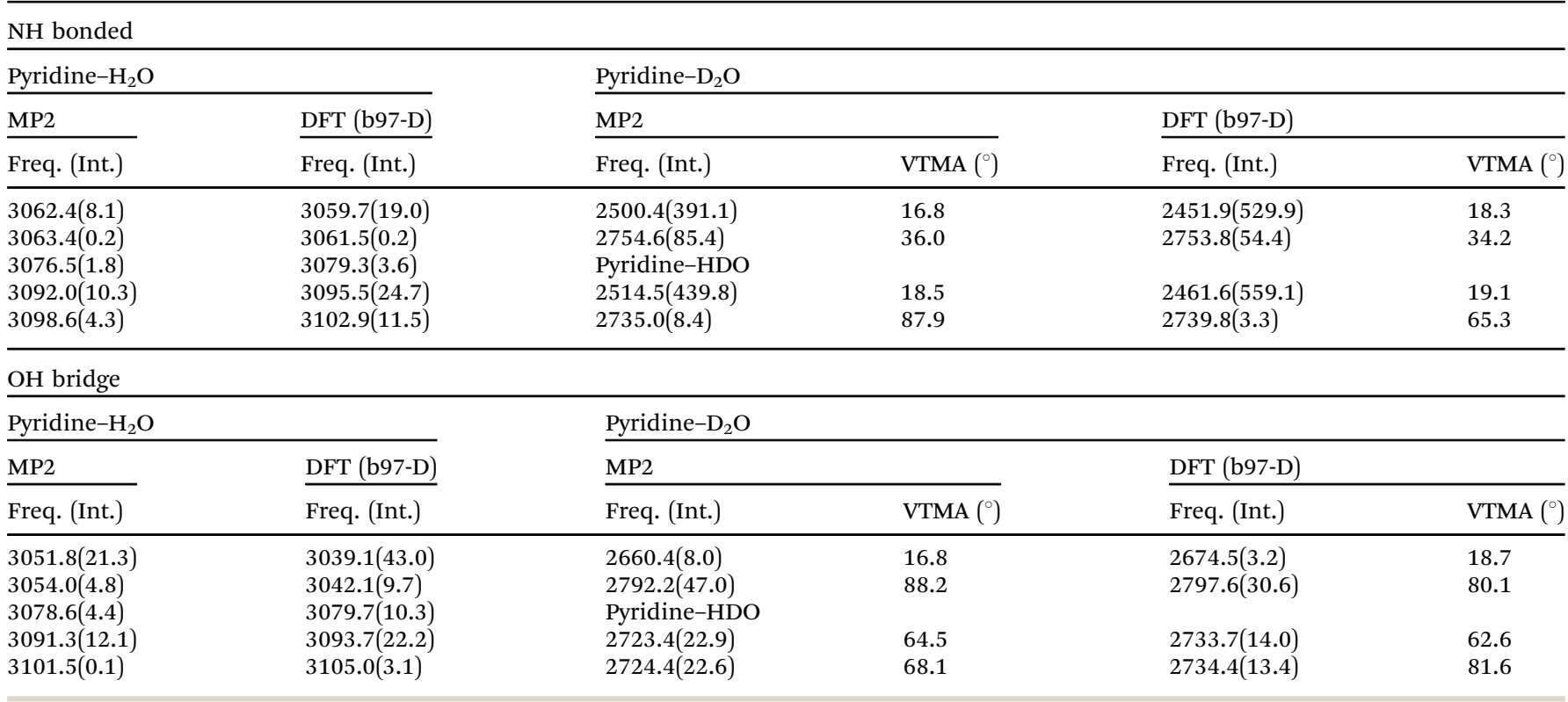

ion current was kept at $1.0 \times 10^{-10} \mathrm{~A}$. We observed bands which were assigned to the vibrational modes of the pyridine monomer and dimer. The addition of water (pressure corresponding to $1.0 \times 10^{-10} \mathrm{~A}$ in the residual gas analyzer) gave rise to additional bands which are shown in Fig. 3 (marked with gray shaded areas).

Bands which have to be assigned to pyridine $-\mathrm{H}_{2} \mathrm{O}$ oligomers were found at $3061.25 \mathrm{~cm}^{-1} ; 3066.90 \mathrm{~cm}^{-1} ; 3076.10 \mathrm{~cm}^{-1}$; $3093.47 \mathrm{~cm}^{-1}$ and $3096.52 \mathrm{~cm}^{-1}$. The two most intense bands were observed at $3076.10 \mathrm{~cm}^{-1}$ and $3093.47 \mathrm{~cm}^{-1}$ while the remaining three bands have significantly lower intensities. An unambiguous assignment is not straight forward, since the predicted absorption bands are very close in frequency. However, the band intensities are not in agreement with the

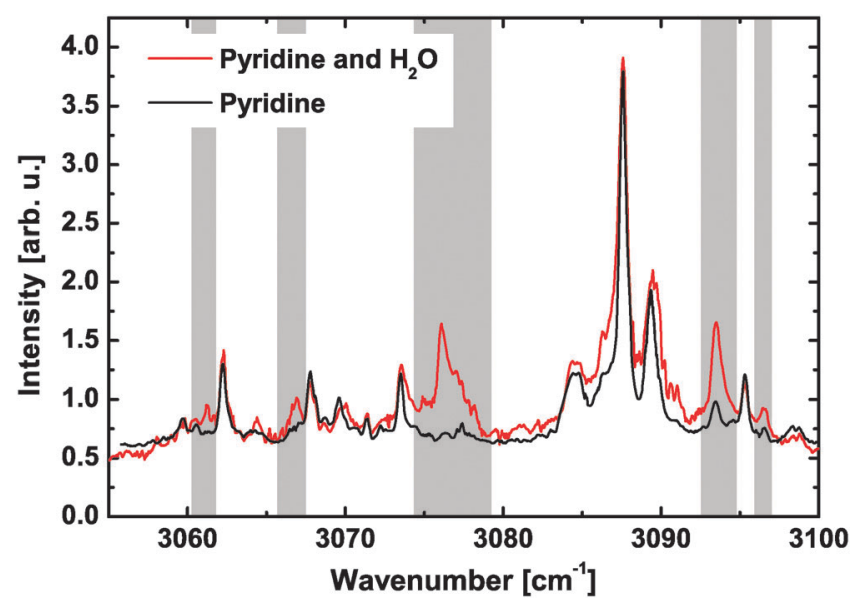

Fig. 3 Depletion spectra of pyridine (black) and pyridine-water (red) complexes in the $\mathrm{C}-\mathrm{H}$ stretch frequency range. The bands attributed to pyridine-water structures are highlighted with gray shaded areas. predictions for the IR bands of the global energy minimum heterodimer. While the predicted intensity ratios are in the range of 5.8-6.9 (depending on the used ab initio method), the observed bands have similar intensities. Furthermore, the asymmetric shape of the band at $3076.10 \mathrm{~cm}^{-1}$ is an indication of the presence of another underlying band, making the intensity determination rather inaccurate. In addition, an unambiguous assignment was hindered by the overlap of the pyridine-water bands with pyridine oligomer bands.

\subsection{IR spectra of pyridine- $\mathrm{D}_{2} \mathrm{O}$ structures in the $\mathrm{D}-\mathrm{O}$ stretch range}

We have recorded the IR spectra in the range from 2650 to $2810 \mathrm{~cm}^{-1}$. In this range, we could detect D-O stretch bands from $\mathrm{D}_{2} \mathrm{O}$ clusters as well as mixed clusters. IR-bands can be attributed to clusters with both pyridine and $\mathrm{D}_{2} \mathrm{O}$ content by a simple method, i.e. the bands were observed to vanish by lowering the partial pressure of both pyridine and $\mathrm{D}_{2} \mathrm{O}$. All bands displayed in Fig. 4 were assigned to pyridine-water oligomers.

In order to assign the bands to a specific (pyridine $)_{n}\left(\mathrm{D}_{2} \mathrm{O}\right)_{m}$ cluster, we have recorded the so-called pick-up curves: we have varied the partial pressure and measured the band intensities as a function of pressure. It is well known that the pick-up of molecules by helium droplets follows a Poisson statistics. ${ }^{13}$ The pick-up curves for bands 1,3 and 5 are plotted in Fig. 5 . It is expected that the pressure values of the maxima of the measured pick-up curve are proportional to the number of pyridine molecules in the cluster responsible for that band. As is evident from Fig. 5, the intensities for bands 3 and 5 have a maximum at about the same pressure while it reaches a maximum at twice that value for band 1 . If we assign the bands 3 and 5 to (pyridine) $-\left(\mathrm{H}_{2} \mathrm{O}\right)_{x}$ then band 1 has to be attributed to (pyridine $)_{2}-\left(\mathrm{H}_{2} \mathrm{O}\right)_{y}$. 


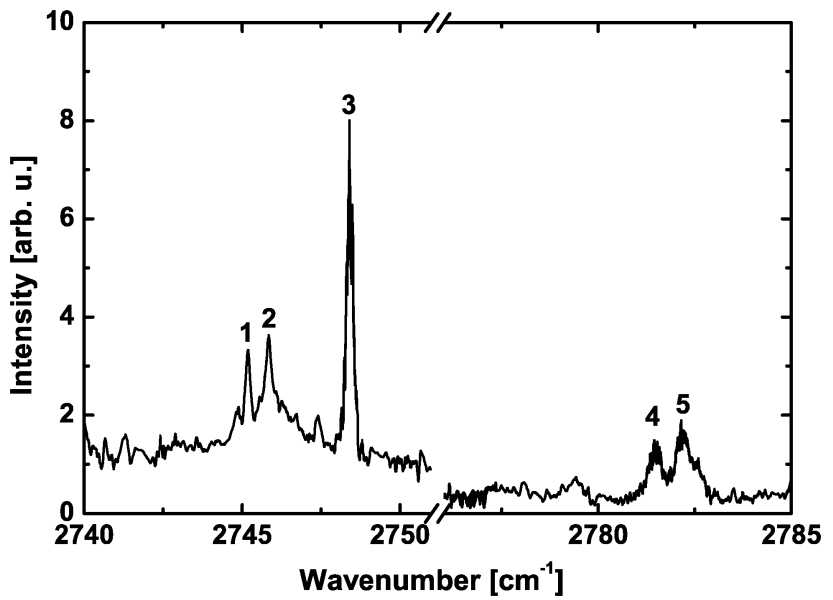

Fig. 4 Bands of (pyridine) ${ }_{n}\left(\mathrm{D}_{2} \mathrm{O}\right)_{m}$ clusters in the $\mathrm{D}-\mathrm{O}$ stretch absorption region.

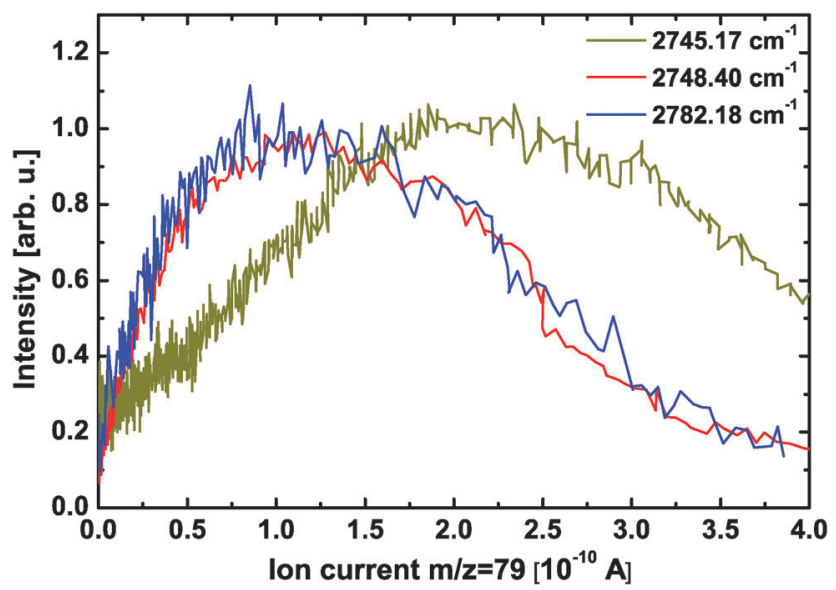

Fig. 5 Pick-up curves for pyridine $-\mathrm{D}_{2} \mathrm{O}$ bands upon variation of the pyridine pressure. Band intensities have been scaled for comparison.

It was not possible to unambiguously determine the number of $\mathrm{D}_{2} \mathrm{O}$ molecules in the same way. As can be seen in Fig. 4, a broad band is present in the frequency range from 2740 to $2750 \mathrm{~cm}^{-1}$. This broad absorption feature was observed to increase in intensity when increasing the $\mathrm{D}_{2} \mathrm{O}$ pick-up pressure. Therefore, a simple pick-up curve would not yield any reliable result since the different contributions to the depletion intensity cannot be disentangled. A different method, used previously by Vilesov and coworkers, ${ }^{24}$ was used instead. We recorded frequency scans for different $\mathrm{D}_{2} \mathrm{O}$ pick-up pressures. By assuming a Poisson statistics, the logarithm of the ratio of two band intensities is given by eqn (1).

$$
\begin{aligned}
\log \left(\frac{I_{k}^{X, Y}}{I_{l}^{M, N}}\right)= & (X-M) \cdot \log \left(Z_{\text {Pyr. }}\right) \\
& +(Y-N) \cdot \log \left(Z_{\mathrm{D}_{2} \mathrm{O}}\right)+\text { const. }
\end{aligned}
$$

where $I_{k}^{X, Y}$ is the intensity of an unassigned band centered at $k$ frequency which is assigned to a cluster with $X$ pyridine molecules and $Y \mathrm{D}_{2} \mathrm{O}$ molecules. $I_{l}^{M, N}$ is the intensity of the reference

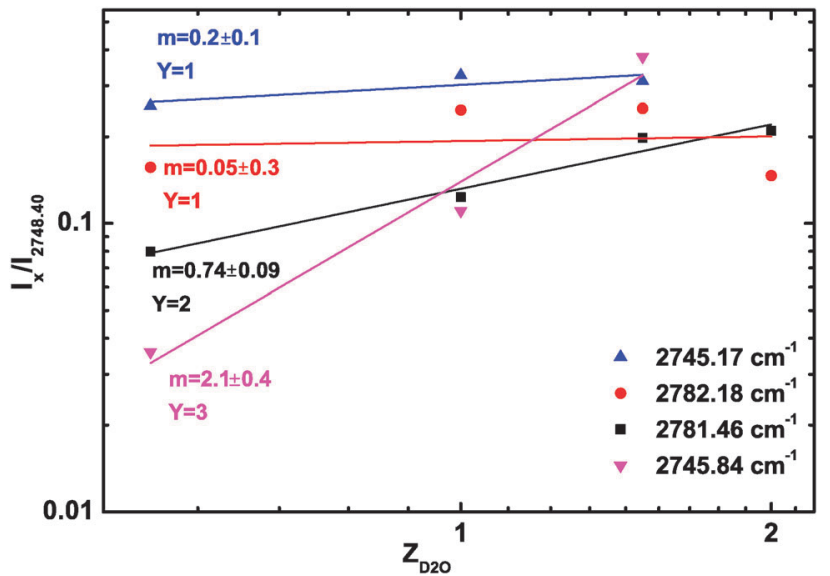

Fig. 6 Intensity ratios for distinct pyridine $-\mathrm{D}_{2} \mathrm{O}$ bands. The results of the fit of the experimental band intensities to eqn (1) are shown as solid lines. The values for the slopes $m$ for the linear fits are given.

band with $M$ pyridines and $N$ deuterated water molecules at frequency 1 . $Z_{\mathrm{Pyr} \text {. }}$ and $Z_{\mathrm{D}_{2} \mathrm{O}}$ are the average numbers of captured pyridine and water molecules, respectively, and are proportional to the pick-up pressures. ${ }^{25}$ The proportionality coefficient for both was obtained from the position of the maximum in the pyridine pick-up curves. The band centered at $2748.40 \mathrm{~cm}^{-1}$ was chosen to be the reference band assuming $M=1$ and $N=1$. The results for the fit of the experimental data to eqn (1) for the different bands are shown in Fig. 6. We find that the bands at 2745.17 and $2782.18 \mathrm{~cm}^{-1}$ show the same dependency within the experimental uncertainty as the reference band and, therefore, are assigned to oligomers with the same number of $\mathrm{D}_{2} \mathrm{O}$ molecules. The results are summarized in Table 2.

As already mentioned, for the $\mathrm{D}_{2} \mathrm{O}$ experiments we expect unavoidable fast exchange reactions taking place with the residual $\mathrm{H}_{2} \mathrm{O}$ present in the gas inlet system. As a result, HDO was also detected in the pick-up chamber in addition to $\mathrm{D}_{2} \mathrm{O}$. For this reason, the HDO pressure at the pick-up chamber was monitored by means of the residual gas analyzer $(\mathrm{m} / \mathrm{z}=19)$ during the experiments. Typically, a $\mathrm{D}_{2} \mathrm{O}: \mathrm{HDO}$ ratio of about $5: 1$ was measured. Hence, the presence of HDO containing oligomers in the experiments can in principle not be excluded. The frequencies of the experimentally observed bands can be compared with the predicted band centers for both conformers, as shown in Table 1 . It can be clearly seen that HDO bands are predicted to have lower intensities compared to those from pyridine- $\mathrm{D}_{2} \mathrm{O}$ heterodimers. In the case of the $\mathrm{NH}$ bounded structure, the IR band intensities of the HDO bands are predicted to be $c a$. 10 times smaller than the $\mathrm{D}_{2} \mathrm{O}$ bands.

Table 2 Fitted numbers of pyridine and $\mathrm{D}_{2} \mathrm{O}$

\begin{tabular}{llll}
\hline No. & Position $\left(\mathrm{cm}^{-1}\right)$ & Pyridine mol. & $\mathrm{D}_{2} \mathrm{O}$ mol. \\
\hline 1 & 2745.17 & 2 & 1 \\
2 & 2745.84 & 1 & 3 \\
3 & 2748.40 & 1 & 1 \\
4 & 2781.46 & 1 & 2 \\
5 & 2782.18 & 1 & 1
\end{tabular}


In addition, the low HDO partial pressure in the pick-up chamber is expected to diminish the bands by a factor of 5 with respect to $\mathrm{D}_{2} \mathrm{O}$ containing bands. Therefore, we expect bands of pyridine-HDO heterodimers to have between one and two orders of magnitude less intensity than those for the $\mathrm{NH}$ bounded pyridine- $\mathrm{D}_{2} \mathrm{O}$ heterodimer bands.

As a result, we assigned bands 3 and 5 to pyridine- $\mathrm{D}_{2} \mathrm{O}$ heterodimers. Considering the band positions, band 3 is assigned to the $\mathrm{NH}$ conformer and band 5 to an IR-band of the $\mathrm{OH}$ bridge conformer (see Table 1). The predicted central frequencies match the pyridine- $\mathrm{D}_{2} \mathrm{O}$ bands within $\pm 10 \mathrm{~cm}^{-1}$. Furthermore, predicted frequency differences between both bands, $37.6 \mathrm{~cm}^{-1}$ for MP2 and $43.8 \mathrm{~cm}^{-1}$ for DFT, are in good agreement with the value $33.78 \mathrm{~cm}^{-1}$ found experimentally.

\subsection{Dependency of the bands on applying a DC electric field}

In order to achieve an unambiguous assignment, additional experiments were carried out to measure the intensity dependence when applying a DC electric field. This technique was applied for the first time in the early 90 's to molecular beams ${ }^{26-}$ ${ }^{28}$ and in 1999 to molecules embedded in helium nanodroplets by Miller and coworkers. ${ }^{29}$ The orientation of the permanent dipole distribution for molecules in a DC electric field can be calculated accurately using the methods described by Kong and coworkers. ${ }^{30,31}$ Under field free conditions, the dipole orientation distributions represent a thermal average over the Stark levels at the rotational temperature of the molecule, i.e. $0.37 \mathrm{~K}$. The Stark energies of the different levels and, hence, the dipole distributions will depend on the permanent dipole and on the rotational constants of the molecule. In the present study, both quantities were obtained from the $a b$ initio calculations. The predicted rotational constants were divided by a factor of three accounting for the fact that a part of the helium density follows the molecular rotation which is a good approximation for molecules with small gas phase rotational constants embedded in helium nanodroplets. ${ }^{11}$ It should also be noted that the dipole distributions depend only weakly on the rotational constants, thus, the error due to this approximation is not expected to be important. In the present system, we made sure that decreasing the rotational constants by a factor of two resulted in relative change of less than $20 \%$ in the calculated field on/field off intensity ratios.

The dipole distribution, $P(\cos \theta)$, for the pyridine-water structures was obtained by carrying out the quantum calculation described elsewhere. ${ }^{32}$ After calculating $P(\cos \theta)$ for a given applied field, the field on/field off intensity ratios for both configurations can be calculated using eqn (2) and (3). The laser polarization axis and the DC electric field orientation were either parallel (eqn (2)) or perpendicular (eqn (3)) to each other.

$$
A_{\|}(\alpha)=2 \int_{0}^{\pi} P(\cos \theta)\left[2 \cos ^{2} \theta+\sin ^{2} \alpha-3 \cos ^{2} \theta \sin ^{2} \alpha\right] \sin \theta \mathrm{d} \theta
$$

$$
A_{\perp}(\alpha)=\int_{0}^{\pi} P(\cos \theta)\left[2-\sin ^{2} \alpha-2 \cos ^{2} \theta+3 \cos ^{2} \theta \sin ^{2} \alpha\right] \sin \theta \mathrm{d} \theta
$$

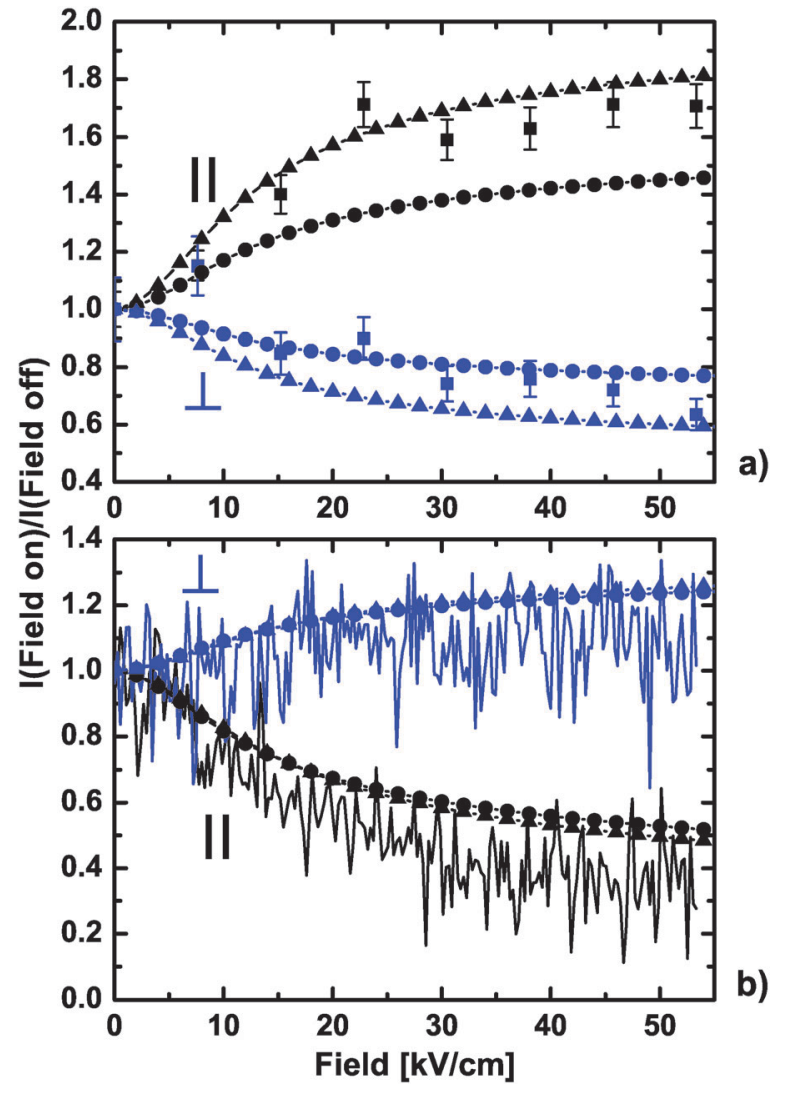

Fig. 7 Field on/field off intensity ratios for the DC electric field oriented perpendicular (blue) or parallel (black) to the laser polarization axis for the pyridine $-\mathrm{D}_{2} \mathrm{O}$ band at $2748.40 \mathrm{~cm}^{-1}$ (a) and at $2782.18 \mathrm{~cm}^{-1}$ (b). Calculations using DFT (triangles) and MP2 (circles) methods are also shown for $\mathrm{NH}$ bonded structure (a) and $\mathrm{OH}$ bridge structure (b).

$\alpha$ is the Vibrational Transition Moment Angle (VTMA) that could be predicted for the different conformer bands based upon the $a b$ initio calculations (Table 1). The field on/field off intensity ratios were measured for bands 3 and 5 . For band 3, the rotational substructure could be partially resolved resulting in a change in the band profile upon applying the DC electric field. Hence, the band profile was measured for different DC field intensities and the IR intensities were integrated over the entire band. For band 5 the rotational substructure was not resolved and the depletion signal at the band center was measured as a function of the field.

The experimental and theoretical results are shown in Fig. 7. As an input we used the predicted VTMA and permanent dipole moments, for the $\mathrm{NH}$ conformer, and compared these results with the experimentally recorded intensity ratios of band 3 . The predictions for the $\mathrm{OH}$ bridge conformer were compared with the experimentally determined intensity dependence of band 5 . In both cases we find a very good agreement between the theoretical predictions and the experimental results confirming our previous assignment. The predictions for the $\mathrm{NH}$ conformer depend on the theoretical method. However, the variation of the predicted VTMA $\left(1.8^{\circ}\right)$ and the predicted permanent electric dipole moments (4.71 D for DFT and 4.22 D for MP2) with 
the level of theory is rather small. The variation in the predicted intensity ratios is rather large due to their nonlinear dependency. It is also worth mentioning that the structures obtained with the two methods differ slightly for the $\mathrm{NH}$ conformer. In the case of the DFT calculation, the structure contains a symmetry plane along the $\mathrm{N} \cdots \mathrm{H}$ axis and perpendicular to the pyridine plane. No symmetry plane is found for the structure shown in Fig. 2(a) obtained using the MP2 method. In this case, the oxygen is displaced towards one of the hydrogen atoms of the ring. Unfortunately, no information on the rotational constants was obtained in the present experiment that would allow us to distinguish between both possibilities.

It is interesting to consider the intensity ratios for the two bands from both conformers. The $\mathrm{NH}$ conformer/OH conformer intensity ratio is 1.8 while in the experiment a value of $5.0 \pm 1.4$ is observed. Therefore, we estimate a population distribution between $\mathrm{NH}$ and $\mathrm{OH}$ bridge structures of $2.8 \pm 0.8$ in helium nanodroplets. From the 1025 structures randomly generated for the minimum search only 72 were found to be minimized to the $\mathrm{OH}$ structure. This corresponds to a ratio of 14.2 between the $\mathrm{NH}$ conformer/OH conformer. Very likely the electrostatic steering plays a role in the aggregation process, favoring the formation of the $\mathrm{OH}$ bridge structure. Further theoretical studies are required to shed some light on this point.

It should be mentioned that we have investigated the possibility of forming a $\pi$ bonded heterodimer in the present experiments. This conformer was found for the benzene-water heterodimer in previous experiments in the gas phase $\mathrm{e}^{33}$ and in the liquid phase. ${ }^{34}$ Using DFT calculations, we found a structure in which one hydrogen is $\mathrm{H}$ bonded to the $\pi$ electron network and the second hydrogen atom of the water molecule is bonded to the nitrogen. However, the expected field dependency for the $\mathrm{D}-\mathrm{O}$ stretch for the band at $2776.2 \mathrm{~cm}^{-1}$ predicted for this structure does not match the experimental results (VTMA 46.1 ${ }^{\circ}$ ). Furthermore, this structure was not found to be an energy minimum at the MP2/aug-cc-pVTZ level of theory. Additional calculations from randomly generated starting configurations using MP2 were also not successful. Therefore, we ruled out the formation of a $\pi$ bonded heterodimer in our experiments.

In all previous experimental studies carried out on pyridine solvated in water ${ }^{35}$ and in the gas phase, ${ }^{36}$ only the global energy minimum structure was considered. Another study of the heterodimer in chloroform solution also found only the $\mathrm{NH}$ conformer. ${ }^{37}$ However, these experiments were carried out at room temperature. Therefore, it is easy to understand the absence of the $\mathrm{OH}$ bridge conformer considering the very low barrier for the conformational rearrangement. A thermal energy corresponding to $105 \mathrm{~K}$ is enough to surmount the barrier which would prevent the observation of the $\mathrm{OH}$ structure. Low temperature (10-12 K) studies using the argon matrix isolation technique were carried out by Maes and coworkers who considered exclusively the NH conformer. ${ }^{23}$ In their work, the splitting in the $\mathrm{OH}$ stretch bands was speculated to originate from matrix shift or by different bonding sites of the water molecule. In the present study, the existence of a new conformer was proposed. Compared to the argon matrix studies reported by Maes and coworkers, the low temperature used in our experiment in combination with the fast cooling rate during aggregation in helium nanodroplets is expected to favor the stabilization of the $\mathrm{OH}$ bridge conformer.

\section{Conclusion}

We report here the IR spectroscopy of pyridine-water heterodimers aggregated in helium nanodroplets. The $\mathrm{C}-\mathrm{H}$ stretch spectral range was measured and five new bands were observed when adding water in the pick-up chamber in addition to pyridine. Further experiments with pyridine $-\mathrm{D}_{2} \mathrm{O}$ in the $\mathrm{D}-\mathrm{O}$ spectral range allowed us to propose the co-existence of two conformers in helium droplets i.e. the $\mathrm{NH}$ bonded and the $\mathrm{OH}$ bridge structures. These results could be confirmed by the application of a DC electric field which allowed us to deduce the angle between the vibrational transition moment and the permanent dipole moment for the different absorption bands. The results were found to be in very good agreement with the results from $a b$ initio predictions. In previous noble gas matrix isolation and liquid phase spectroscopy studies only the $\mathrm{NH}$ bonded structure was observed. The present results show that the $\mathrm{OH}$ bridge structure can be formed in the pyridine-water heterodimer in helium nanodroplets. This can be explained by taking into consideration that helium nanodroplets can stabilize structures which correspond to local minima of the potential energy surface.

\section{Acknowledgements}

Support by DFG (Grants HA 2394/13) within FOR 618 (Molecular Aggregation) is gratefully acknowledged as well as computer resources from BOVILAB@RUB and Rechnerverbund-NRW. P.N. thanks the Marie Curie Program for financial support. This work is part of the Cluster of Excellence RESOLV (EXC 1069) funded by DFG.

\section{References}

1 G. C. Pimentel and A. L. McClellan, The Hydrogen Bond, Freemand and Co., San Francisco, 1960.

2 G. A. Jeffrey and W. Saenger, Hydrogen Bonding in Biological Structures, Springer-Verlag, Berlin, 1991.

3 M. Behrens, U. Buck, R. Frochtenicht, M. Hartmann and M. Havenith, J. Chem. Phys., 1997, 107, 7179-7186.

4 F. Madeja, M. Havenith, K. Nauta, R. E. Miller, J. Chocholousova and P. Hobza, J. Chem. Phys., 2004, 120, 10554-10560.

5 M. Ortlieb, O. Birer, M. Letzner, G. W. Schwaab and M. Havenith, J. Phys. Chem. A, 2007, 111, 12192-12199.

6 A. Buckingham, J. D. Bene and S. McDowell, Chem. Phys. Lett., 2008, 463, 1-10.

7 A. Gutberlet, G. Schwaab and M. Havenith, J. Phys. Chem. A, 2011, 115, 6297-6305.

8 J. E. Del Bene, J. Am. Chem. Soc., 1975, 97, 5330-5335.

9 G. Maes, Bull. Soc. Chim. Belg., 1981, 90, 1093-1107. 
10 J. C. Howard, N. I. Hammer and G. S. Tschumper, ChemPhysChem, 2011, 12, 3262-3273.

11 M. Y. Choi, G. E. Douberly, T. M. Falconer, W. K. Lewis, C. M. Lindsay, J. M. Merritt, P. L. Stiles and R. E. Miller, Int. Rev. Phys. Chem., 2006, 25, 15-75.

12 J. P. Toennies and A. F. Vilesov, Angew. Chem., Int. Ed., 2004, 43, 2622-2648.

13 M. Lewerenz, B. Schilling and J. P. Toennies, J. Chem. Phys., 1995, 102, 8191-8207.

14 K. Nauta and R. E. Miller, Science, 1999, 283, 1895-1897.

15 K. Nauta and R. E. Miller, Science, 2000, 287, 293-295.

16 G. O. Sorensen, L. Mahler and N. Rastrup-Andersen, J. Mol. Struct., 1974, 20, 119-126.

17 S. A. Clough, Y. Beers, G. P. Klein and L. S. Rothman, J. Chem. Phys., 1973, 59, 2254-2259.

18 K. von Haeften, S. Rudolph, I. Simanovski, M. Havenith, R. E. Zillich and K. B. Whaley, Phys. Rev. B: Condens. Matter Mater. Phys., 2006, 73, 054502.

19 M. Lewerenz, B. Schilling and J. P. Toennies, Chem. Phys. Lett., 1993, 206, 381-387.

20 J.-S. Samson, G. Wollny, E. Bründermann, A. Bergner, A. Hecker, G. Schwaab, A. D. Wieck and M. Havenith, Phys. Chem. Chem. Phys., 2006, 8, 753-758.

21 TURBOMOLE V6.4 2013, a development of University of Karlsruhe and Forschungszentrum Karlsruhe $\mathrm{GmbH}$, 1989-2007, TURBOMOLE GmbH, since 2007; available from http://www.turbomole.com.

22 S. Grimme, J. Antony, S. Ehrlich and H. Krieg, J. Chem. Phys., 2010, 132, 154104.
23 A. Destexhe, J. Smets, L. Adamowicz and G. Maes, J. Chem. Phys., 1994, 98, 1506-1514.

24 S. D. Flynn, D. Skvortsov, A. M. Morrison, T. Liang, M. Y. Choi, G. E. Douberly and A. F. Vilesov, J. Phys. Chem. Lett., 2010, 1, 2233-2238.

25 M. N. Slipchenko, K. E. Kuyanov, B. G. Sartakov and A. F. Vilesov, J. Chem. Phys., 2006, 124, 241101.

26 B. Friedrich and D. Herschbach, Z. Phys. D: At., Mol. Clusters, 1991, 18, 153-161.

27 H. J. Loesch and A. Remscheid, J. Chem. Phys., 1990, 93, 4779-4790.

28 J. M. Rost, J. C. Griffin, B. Friedrich and D. R. Herschbach, Phys. Rev. Lett., 1992, 68, 1299-1302.

29 K. Nauta and R. E. Miller, Phys. Rev. Lett., 1999, 82, 4480-4483.

30 W. Kong, Int. J. Mod. Phys. B, 2001, 15, 3471.

31 W. Kong and J. Bulthuis, J. Phys. Chem. A, 2000, 104, 1055-1063.

32 M. Y. Choi and R. E. Miller, J. Am. Chem. Soc., 2006, 128, 7320-7328.

33 S. Suzuki, P. G. Green, R. E. Bumgarner, S. Dasgupta, W. A. Goddard and G. A. Blake, Science, 1992, 257, 942-945.

34 K. P. Gierszal, J. G. Davis, M. D. Hands, D. S. Wilcox, L. V. Slipchenko and D. Ben-Amotz, J. Phys. Chem. Lett., 2011, 2, 2930-2933.

35 H. Takahashi, K. Mamola and E. K. Plyler, J. Mol. Spectrosc., 1966, 21, 217-230.

36 D. J. Millen and G. Mines, J. Chem. Soc., Faraday Trans., 1977, 73, 369.

37 N. Soon, Spectrochim. Acta, Part A, 1979, 35, 515-516. 